\title{
iFeel_IM! Emotion Enhancing Garment for Communication in Affect Sensitive Instant Messenger
}

\author{
Dzmitry Tsetserukou ${ }^{1}$, Alena Neviarouskaya ${ }^{2}$, Helmut Prendinger ${ }^{3}$, \\ Naoki Kawakami ${ }^{1}$, Mitsuru Ishizuka ${ }^{2}$, and Susumu Tachi ${ }^{1}$ \\ ${ }^{1}$ Department of Information Physics and Computing, University of Tokyo, Japan \\ dima_teterukovaipc.i.u-tokyo.ac.jp, \\ \{kawakami, tachi\}astar.t.u-tokyo.ac.jp \\ ${ }^{2}$ Department of Information and Communication Engineering, University of Tokyo, Japan \\ lena@mi.ci.i.u-tokyo.ac.jp, ishizuka@i.u-tokyo.ac.jp \\ ${ }^{3}$ National Institute of Informatics, Japan \\ helmut@nii.ac.jp
}

\begin{abstract}
In the paper we are proposing a conceptually novel approach to reinforcing (intensifying) own feelings and reproducing (simulating) the emotions felt by the partner during online communication through specially designed system, iFeel_IM!. The core component, Affect Analysis Model, automatically sense nine emotions from text. The detected emotion is stimulated by innovative haptic devices (HaptiHeart, HaptiHug, HaptiTickler, HaptiCooler, and HaptiWarmer) integrated into iFeel_IM! The implemented system can considerably enhance emotionally immersive experience of real-time messaging.
\end{abstract}

Keywords: Affective haptics, affective user interface, wearable devices.

\section{Introduction}

"All emotions use the body as their theater..." Antonio Damasio [1] Pleasure, euphoria, sadness, fear, anger - these and other emotions color our lives, enrich our experiences, and imbue our actions with passion and character. Research on emotion in both psychology and neuroscience indicates that emotions also play a pivotal role in social interaction, decision-making, perception, attention, memory, learning, etc. Affective Computing [2], which aims to realize emotionally intelligent human-computer interaction, is a well-established research area that deals with investigation of the role of emotions in technology and poses a question: "How can we make computer to be aware of emotions?" While conducting our research in an emerging frontier of Affective Haptics, we are attempting to answer another question: "How to elicit (or enhance) the user's affective state in human-human mediated communication by haptic technologies?"

Besides emotions conveyed through text, researchers developed an additional modality for communicating emotions in Instant Messenger (IM) through tactile interfaces with vibration patterns $[3,4,5,6]$. However, in the proposed methods users 
have to memorize the vibration or pin matrix patterns and cognitively interpret the communicated emotional state. Demodulation of haptically coded emotion is not natural for human-human communication, and direct evocation of emotion cannot be achieved in such system.

Driven by the motivation to enhance social interactivity and emotionally immersive experience of real-time messaging, we are proposing a conceptually novel approach to reinforcing (intensifying) own feelings and reproducing (simulating) the emotions felt by the partner through specially designed system, iFeel_IM!. The philosophy behind the iFeel_IM! (intelligent system for Feeling enhancement powered by affect sensitive Instant Messenger) is "I feel [therefore] I am!". The emotion evoked by physical stimulation might imbue our communication with passion and increase the emotional intimacy, ability to be close, loving, and vulnerable. We argue that interpersonal relationship and the ability to express empathy grow strongly when people become emotionally closer through disclosing thoughts, feelings and emotions for the sake of understanding.

In this work, we focus on implementation of innovative system and devices for generation of physical stimulation aimed to convey the emotion experienced during online conversations. We attempt to influence on human emotions by physiological changes, physical stimulation, and social touch.

\subsection{Physiological Changes Evoke Human Emotions}

Our emotional states include two components: physical sensations (heart rate, respiration, etc.) and conscious feelings (joy, anger, etc.). Emotional states are mediated by a family of peripheral, autonomic, endocrine, and skeletomotor responses. When frightened, we not only feel afraid but also experience increased heart rate and respiration, dryness of the mouth, tense muscles, and sweaty palms [7].

According to James-Lange theory [8], the conscious experience of emotion occurs after the cortex receives signals about changes in physiological state. Researchers argued that feelings are preceded by certain physiological changes. Thus, when we see a venomous snake, we feel afraid because our cortex has received signals about our racing heart, knocking knees, etc. In [9], Prinz revived the James-Lange theory, supported it with reference to recent empirical research, and thoroughly criticized cognitive theories of emotions for downplaying the role of the body.

Among a variety of physiological changes accompanying the experience of emotion (heart rate, temperature, respiration, pulse, blood pressure, muscle action potentials), for our research we selected those (heart rate and temperature) that can be affected externally in a safe manner. The ability of false heart rate feedback to change our emotional state was reported in [10]. Briese [11] found a tight relationship between emotions and body temperature.

To our knowledge, the only work concentrating on the emotion induction through physical means is described in [12]. The interactive chair changes emotional state of the subject watching the movie with rich emotional content. However, user study did not show any significant effect of the chair on intensity of experienced emotion. The reported reasons are: (1) weakness of the technical solutions (in many cases users did not perceive any physical influence, and complained on interruption effect on the movie watching); (2) user-study procedure was not well elaborated. 


\subsection{Mediated Social Touch and Haptic Communication of Emotions}

In a real world, whenever one person interacts with another, both observe, perceive and interpret each other's emotional expressions communicated through a variety of signals. Valuable information is also transferred by non-verbal communication (e.g. social touch). It is well known that touching is one of the most powerful means for establishing and maintaining social contact. The fact that two people are willing to touch implies an element of trust [13]. Expressive potential of touch is the ability to convey and elicit strong emotions. Although current on-line interaction mainly relies on senses of vision and hearing, there is a substantial need in mediated social touch. Comprehensive review of research on social touch is presented in [14].

Among many forms of physical contact, the most emotionally charged one is hug. It conveys warmth, love, and affiliation. The Hug Shirt allows people who are missing each other to send physical sensation of the hug over distance [15]. User can wear this shirt, embedded with actuators and sensors, in everyday life. The communication is performed through cell phone facilitated with Bluetooth and Java software. DiSalvo et al. [16] devised "The Hug" interface. When person desires to communicate hug, he/she squeezes the pillow that results in vibration and temperature changes in the partner's device.

Even though such devices can potentially increase interactivity, they have such drawbacks as: (1) the functionality is highly limited and is not extendable (only hug pattern can be transmitted); (2) inconvenient design (users found them difficult to use in real life); (3) inability to resemble natural hug sensation and, hence, elicit strong affective experience (only slight pressure is generated by vibration actuators); (4) lack of visual representation of the partner.

We believe that it is possible to highly increase the emotional immersion through the iFeel_IM! system featuring automatic affect recognition from text, visualization, and physical stimulation feedback.

\section{2 iFeel_IM! Architecture}

Nowadays, communication through IM and chat is very popular. However, during online communication people are concentrating on textual information and are only slightly affected emotionally. Conventional mediated systems usually (1) support only simple textual cues like emoticons; (2) lack visual emotional signals such as facial expressions and gestures; (3) support only manual control of expressiveness of graphical representations of users (avatars); (4) completely ignore such important channel of social communication as sense of touch.

In the iFeel_IM! system, great importance is placed on the automatic sensing of emotions conveyed through textual messages, visual reflection of the detected emotions and communicative behaviour through a 2D cartoon-like avatars, enhancement of user's affective state, and reproduction of feeling of social touch (e.g., hug) by means of haptic stimulation in a real world. The architecture of the iFeel_IM! system is presented in Fig. 1. 


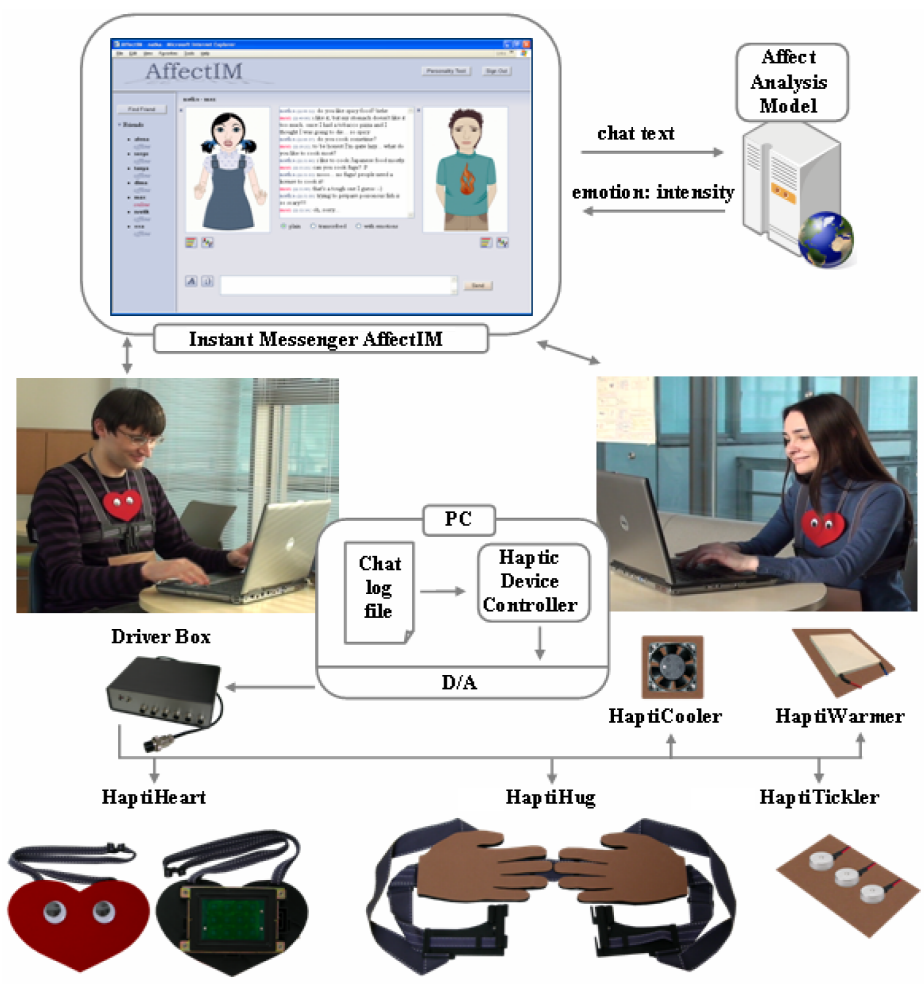

Fig. 1. Architecture of iFeel_IM! system

In order to communicate through iFeel_IM! system, users have to wear innovative haptic devices (HaptiHeart, HaptiHug, HaptiTickler, HaptiCooler, and HaptiWarmer) developed by us.

As a media for communication, we developed AffectIM [17] instant messenger. AffectIM receives the typed utterance, sends it to the Affect Analysis Model [18] responsible for recognition of emotions conveyed through text and located on the server, gets the result (dominant emotion and intensity for each sentence), and visually reflects the sensed affective state through avatar facial expression and gestures.

The results from the Affect Analysis Model are stored along with chat messages in a file on local computer of each user. Haptic Device Controller analyses these data in a real time and generates control signals for Digital/Analog converter (D/A), which then feeds Driver Box for haptic devices with control cues. Based on the transmitted signal, the corresponding haptic device (HaptiHeart, HaptiHug, HaptiTickler, HaptiCooler, or HaptiWarmer) worn by user is activated.

\section{AffectIM: Intelligent Affect Sensitive Instant Messenger}

AffectIM, supported by the Affect Analysis Model, was developed as a web-based application running in the Internet browser. 
For affect categorization, we have decided to use the subset of emotional states defined by Izard [19]: 'anger', 'disgust', 'fear', 'guilt', 'interest', 'joy', 'sadness', 'shame', and 'surprise'. While constructing our Affect Analysis Model we took into account crucial aspects of informal online conversation such as its specific style and evolving language. The affect sensing algorithm consists of five main stages: (1) symbolic cue analysis; (2) syntactical structure analysis; (3) word-level analysis; (4) phrase-level analysis; and (5) sentence-level analysis. Analyzing each sentence in sequential stages, our method is capable of processing sentences of different complexity, including simple, compound, complex (with complement and relative clauses), and complex-compound sentences (details are given in [18]). The working flow of the Affect Analysis Model is presented in Fig. 2.

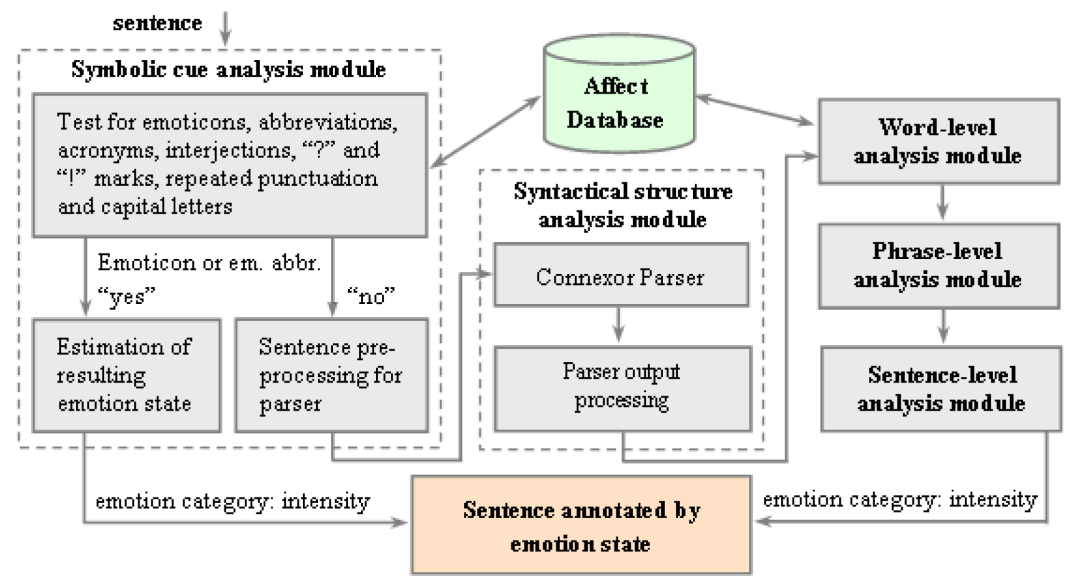

Fig. 2. Working flow of the Affect Analysis Model

An empirical evaluation of the Affect Analysis Model algorithm showed promising results regarding its capability to accurately classify affective information in text from an existing corpus of informal online communication. In a study based on blog entries, the system result agreed with at least two out of three human annotators in $70 \%$ of the cases.

The example of conversation in AffectIM (with emotion annotations from Affect Analysis Model) is given below:

\footnotetext{
A: Hi, good to see you there! [JOY]

$\mathrm{B}: \mathrm{Hi}$, it is great that AffectIM eliminates spacial distance and connects us. [SURPRISE]

A: Right \{\} [HUG]

B: Oh, I really feel the warmth of your hug!!

A: Yes, it's so exciting, as if we met in a real world. [INTEREST]

B: How is your study at the University of Tokyo going on?

A: I have successfully graduated last month. [JOY]

B: Congratulations, I am very proud of you! [JOY]

A: My mind is now filled with pleasant memories about student life, but at the same time I regret this period, full of fun, flew away so fast. [GUILT]
} 
B: You certainly deserve rest.

A: Former students of our Lab have invited me to the trip to Ski resort.

B: I remember the first time I tried to balance on a snowboard was definitely a very thrilling experience! Have you ever been descending a steep slope on a snowboard? [FEAR]

A: I always feel angry at myself for not having enough courage for snowboarding. Shamefully, I prefer safe skiing. [ANGER, SHAME]

B: Sure, extreme sport needs thorough training. My first attempt resulted in a broken leg. But now my experience and protective gear allow me to enjoy snowboarding. [SADNESS, JOY]

A: For me, risky snowboarding might be safe only in a virtual world. :) [JOY]

The twenty-user study conducted on AffectIM [17] showed that the IM system with automatic emotion recognition function was successful at conveying users' emotional states, thus enriching expressivity and social interactivity of online communications.

\section{Affective Haptic Devices}

In order to support the affective communication, we implemented several novel haptic gadgets embedded in iFeel_IM!. They make up three groups. First one is intended for emotion elicitation implicitly (HaptiHeart, HaptiCooler, HaptiWarmer), second type evokes affect in a direct way (HaptiTickler), and third one uses sense of social touch (HaptiHug) for mood influence. All these devices produce a sense of touch including kinesthetic and coetaneous channels. Kinesthetic stimulations, produced by forces exerted on body, are sensed by mechanoreceptors in the tendons and muscles [7]. This channel is highly involved in sensing the stimulus produced by HaptiHug device. On the other hand, mechanoreceptors in the skin layers are responsible for cutaneous stimulation perception. Different types of tactile corpuscles allow us sensing thermal property of the object (HaptiCooler, HaptiWarmer), pressure (HaptiHeart, HaptiTickler), vibration frequency (HaptiTickler), and stimuli location (localization of stimulating device position enables association with particular physical contact).

\subsection{HaptiHug: The Way of Realistic Hugging over Distance}

When people are hugging, they generate pressure on the back of each other by hands, and on the chest area simultaneously. The key feature of the developed HaptiHug is that it physically reproduces the hug pattern similar to that of human-human interaction. The hands for a HaptiHug are sketched from a real human and made from soft material. The important point is that the hands were designed in such a way that the user feels as if the friend's hands actually contact him. Couple of oppositely rotating motors are incorporated into the holder placed on the user's chest area. Soft Hands, aligned horizontally, contact back of the human. Shoulder strips, supporting motor holder and Soft Hands, allow aligning vertical position of the device on human torso. Once 'hug' command is received, couple of motors tense the belt, pressing thus hands and breast part of HaptiHug in direction of human body (Fig. 3). 


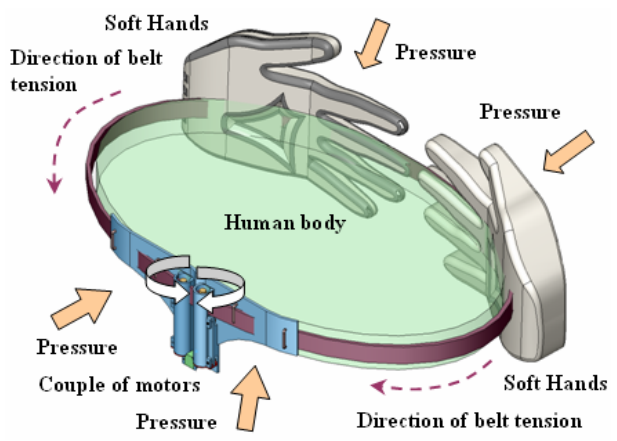

Fig. 3. Structure of wearable HaptiHug device

The duration and intensity of the hug is controlled by the software according to the detected emoticon or keyword. The Driver Box regulates the current magnitude in DC motor from $0 \mathrm{~mA}$ to $500 \mathrm{~mA}$. For presentation of plain hug level (e.g., ' $\left(>^{\wedge} \_\wedge\right)>$ ', ' \{\} ', '<h>'), big hug level (e.g., '>:D<', ' $\{\{\}\}$ '), and great big hug level (e.g., 'gbh', ' $\{\{\{\}\}\}$ '), the pressure of $200 \mathrm{~N} / \mathrm{m}$ with duration of $2 \mathrm{sec}$, the pressure of $300 \mathrm{~N} / \mathrm{m}$ with duration of $3 \mathrm{sec}$, and the pressure of $450 \mathrm{~N} / \mathrm{m}$ with duration of $4 \mathrm{sec}$, was applied on the user's back and chest, respectively.

The technical specification of the device is as follows. DC motors RE $101.5 \mathrm{~W}$ in combination with planetary gearhead GP 10 A (gear ratio of 64:1) generate stall torque of $192.6 \mathrm{mNm}$. The pressure produced on the human chest by tensed belt equals $450 \mathrm{~N} / \mathrm{m}$ at the most. Motor holder was manufactured on 3D printer Dimension DS 768 from ABS plastic material. Special slots were provided to make the pass for pressing belt.

Preliminary, Soft Hands were printed on 3D printer from ABS with thickness of 1 $\mathrm{mm}$. However, plate flexibility was not enough to achieve touch sensation similar to human hand. Therefore, we decided to make hands from compliant rubber-sponge material. The contour profile of Soft Hand is sketched from the male human and has front-face area of $155.6 \mathrm{~cm}^{2}$. Two identical pieces of Soft Hand of $5 \mathrm{~mm}$ thickness were sandwiched by narrow belt slots and connected by plastic screws. Such structure provides enough flexibility to tightly fit to the human back surface while being pressed by belt. Moreover, belt can loosely move inside the Soft Hands during tension period. The structure of HaptiHug is presented in Fig. 4.
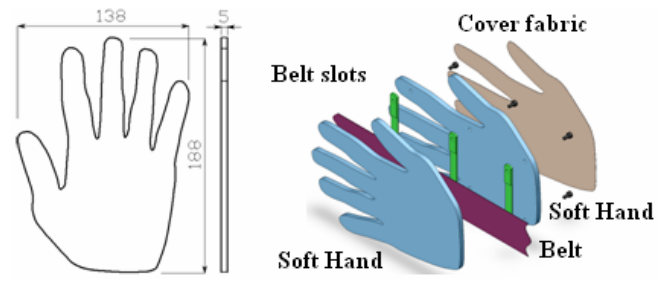

Fig. 4. Structure of Soft Hands 
HaptiHug device has lightweight tri-glide bucklers and side release fastener integrated into the motor holder to facilitate easy adjustment of the belt size to her/his body sizes and detaching it in a natural and rapid manner.

\subsection{HaptiHeart, HaptiCooler, HaptiWarmer, HaptiTickler. Or How We Can Enhance and Influence on Our Emotions by Haptics}

Each emotion is characterized by a specific pattern of physiological changes. Fear features cold, sweaty hands, tensed muscles, increased heart rate, joy - warm hands, increased heart rate. We selected four distinct emotions with strong physical features: 'anger', 'fear', 'sadness', and 'joy'.

There is no doubt that feelings are intuitively connected with the heart, and our lexicon confirms this. The research on interplay between heart rate and emotions revealed that different emotions are associated with distinct patterns of heart rate variations [20]. We developed heart imitator HaptiHeart to produce special heartbeat patterns according to emotion to be conveyed or elicited (sad associated with slightly intense heartbeat, anger with quick and violent heartbeat, fear with intense heart rate).

We take advantage of the fact that our heart naturally synchronizes with the heart of a person we hold or hug. Thus, the heart rate of a user is influenced by haptic perception of the beat rate of the HaptiHeart. Furthermore, false heart beat feedback can directly be interpreted as real heart beat, so it can change the emotional perception.

The HaptiHeart consists of two modules: flat speaker FPS 0304 and speaker holder. The flat speaker sizes $(66.5 \times 107 \times 8 \mathrm{~mm})$ and rated input power of $10 \mathrm{~W}$ allowed us to design powerful and relatively compact HaptiHeart device producing realistic heartbeating sensation with high fidelity. The 3D model is presented in Fig. 5.

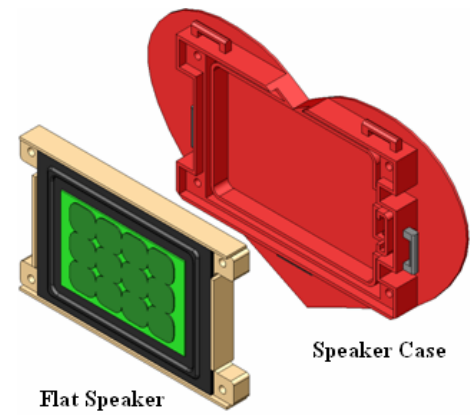

Fig. 5. HaptiHeart layout

We recorded the heartbeat patterns for the cases of experiencing fear, anger, and sadness. The pre-recorded sound signal with low frequency generates the pressure on the human breast through vibration of the speaker surface.

HaptiTickler is responsible for joy emotion evocation. The idea behind this device is to reproduce effect of "Butterflies in the stomach" (fluttery or tickling feeling in the stomach felt by people experiencing love) by means of circular arrays of vibration motors attached to user abdomen area. 
HaptiWarmer is intended for simulation of warmth on the human skin to evoke either aggression or pleasant feeling. It was proved that uncomfortably hot temperatures arouse anger feelings [21]. On the other hand, temperatures within the comfortable region can elicit positive emotions. Based on this evidence, we change the temperature of Peltier element inside HaptiWarmer to be in the limits of $25-36{ }^{\circ} \mathrm{C}$ in the case of joy and temperature range of $40-50{ }^{\circ} \mathrm{C}$ for influencing on anger state. With motivation to boost the fear, we designed HaptiCooler that produces "Coldness on back" effect simulated by the cold airflow along with cold side of Peltier element.

\section{Conclusion and Future Research}

In a nutshell, while developing the iFeel_IM! system, we attempted to bridge the gap between mediated and face-to-face communications by enabling and enriching the spectrum of senses such as vision and touch along with cognition and inner personal state. In the paper we described the architecture of iFeel_IM! and development of novel haptic devices, such as HaptiHeart, HaptiHug, HaptiTickler, HaptiCooler, and HaptiWarmer. These devices were designed with particular emphasis on natural and realistic representation of the physical stimuli, modular expandability, and ergonomic human-friendly design. User can perceive the intensive emotions during online communication, use desirable type of stimuli, comfortably wear and easily detach devices from torso. We are planning to conduct the user study experiments to estimate the performance of the developed system.

On-line communicating users are usually invisible for each other (non-verbal channel is deactivated), and can dissemble actual emotions. This can lead to confusion during text messaging. The iFeel_IM! system can make communication emotionally truthful, so that users will tend to express emotions they actually perceive. It should be noted, that our system can not only enhance the emotional state of the user but also generate emotional feedback (empathy) in communicating person. In future research, we will investigate the topic "how can we make the partner to feel our own emotions". We believe that iFeel_IM! will encourage users to get in "touch" with their emotions and to make social contact with other people in on-line communication richer and more enjoyable.

Computer gaming is very interesting application area for affective haptic devices. The success of Wii has demonstrated that better graphics alone (PS3, Xbox360) is not the key to the game success. Its popularity is mainly due to high involvement of the user into the game through interactivity. By using kinaesthetic and tactile senses with Wii controller, players experience greater physiological arousal. We are planning to design two types of interactive affective game with emotional feedback to create fantastic and emotionally charged playing experiences: (1) player goal is to change emotional state of the virtual characters by performing social touch actions (hugging, patting on the back, handshaking, etc.); (2) during on-line communication players attempt to convey own emotions to partner.

Acknowledgments. The research is supported in part by a Japan Society for the Promotion of Science (JSPS) Postdoctoral Fellowship for Foreign Scholars. 


\section{References}

1. Damasio, A.: The Feeling of What Happens: Body, Emotion and the Making of Consciousness. Vintage, London (2000)

2. Picard, R.W.: Affective Computing. MIT Press, Cambridge (2000)

3. MacLean, K., Enriquez, M.: Perceptual Design of Haptic Icons. In: EuroHaptics, pp. 351363 (2003)

4. Shin, H., Lee, J., Park, J., Kim, Y., Oh, H., Lee, T.: A Tactile Emotional Interface for Instant Messenger Chart. In: 12th International Conference on Human-Computer Interaction, pp. 166-175. Springer, Heidelberg (2007)

5. Rovers, A.F., Van Essen, H.A.: HIM: A Framework for Haptic Instant Messaging. In: 26th SIGCHI Conference on Human Factors in Computing Systems, pp. 1313-1316. ACM, New York (2004)

6. Mathew, D.: vSmileys: Imagine Emotions through Vibration Patterns. In: Alternative Access: Feeling and Games (2005)

7. Kandel, E.R., Schwartz, J.H., Jessel, T.M.: Principles of Neural Science. McGraw-Hill, New-York (2000)

8. James, W.: What is an Emotion? Mind 9, 188-205 (1884)

9. Prinz, J.J.: Gut Reactions: A Perceptual Theory of Emotion. Oxford University Press, Oxford (2004)

10. Decaria, M.D., Proctor, S., Malloy, T.E.: The Effect of False Heart Rate Feedback on Self Reports of Anxiety and on Actual Heart Rate. Behavior research \& Therapy 12, 251-253 (1974)

11. Briese, E.: Emotional Hyperthermia and Performance in Humans. Physiology \& Behavior 58(3), 615-618 (1995)

12. Kok, R., Broekens, J.: Physical Emotion Induction and Its Use in Entertainment: Lessons Learned. In: 1st IFIP Entertainment Computing Symposium, pp. 33-48. Springer, Heidelberg (2008)

13. Collier, G.: Emotional Expression. Lawrence Erlbaum Associates Inc., New Jersey (1985)

14. Haans, A., Jsselsteijn, W.I.: Mediated Social Touch: A Review of Current Research and Future Directions. Virtual Reality 9, 149-159 (2006)

15. Hug Shirt. CuteCircuit Company, http://www. cutecircuit.com

16. DiSalvo, C., Gemperle, F., Forlizzi, J., Montgomery, E.: The Hug: An Exploration of Robotic Form for Intimate Communication. In: 12th IEEE International Workshop on Robot and Human Interactive Communication, pp. 403-408. IEEE Press, New-York (2003)

17. Neviarouskaya, A., Prendinger, H., Ishizuka, M.: User Study of AffectIM, an Emotionally Intelligent Instant Messaging System. In: Prendinger, H., Lester, J.C., Ishizuka, M. (eds.) IVA 2008. LNCS, vol. 5208, pp. 29-36. Springer, Heidelberg (2008)

18. Neviarouskaya, A., Prendinger, H., Ishizuka, M.: Textual Affect Sensing for Sociable and Expressive Online Communication. In: Paiva, A.C.R., Prada, R., Picard, R.W. (eds.) ACII 2007. LNCS, vol. 4738, pp. 220-231. Springer, Heidelberg (2007)

19. Izard, C.E.: Human Emotions. Plenum Press, New York (1977)

20. Anttonen, J., Surakka, V.: Emotions and Heart Rate while Sitting on a Chair. In: 27th SIGCHI Conference on Human Factors in Computing Systems, pp. 491-499. ACM, New York (2005)

21. Anderson, C.A.: Temperature and Aggression: Ubiquitous Effects of Heat on Occurrence of Human Violence. Psychological Bulletin 106(1), 74-96 (1989) 